\title{
Using the Hair Removal Laser in the Axillary Region and its Effect on Normal Microbial Flora
}

\section{Zeynab Fazel', Ali Majidpour², Elham Behrangi ${ }^{*}$, Sara Fathizadeh², Maryam Nokandeh${ }^{1}$, Najmolsadat Atefi', Mohammad Reza Ghassemi ${ }^{1}$}

'Department of Dermatology, Rasol-e-Akram Hospital, Iran University of Medical Sciences, Tehran, Iran

${ }^{2}$ Antimicrobial Research Center, Institute of Immunology Infectious Diseases, Iran University of Medical Sciences, Tehran, Iran

\section{*Correspondence to}

Elham Behrangi, Associate

Professor, Department of Dermatology, Rasol-e-Akram Hospital, Iran University of Medical Science, Tehran, P.O. Box: 1449614535 , Iran; Tel: +98-21- 64352528; Fax: +98-21- 64352528; Email: behrangi.e@iums.ac.ir

Published online June 21, 2020

\begin{abstract}
Introduction: The axillary hair removal laser is one of the most often used procedures to treat unwanted hairs in that region. Employing this technology can be helpful in decreasing the bromhidrosis.

Methods: In the present research, a clinical trial study over the effect of the hair removal laser on normal microbial flora at the axillary region is presented. The intervention group consisted of 30 women referred to the dermatologic clinic for the purpose of removing axillary hair by the alexandrite $755 \mathrm{~nm}$ laser and the control group consisted of 30 women referred to the same clinic for any other reasons. Both groups were evaluated for the type of bacterial strains on the first visit and after three and six months.

Results: The results showed that the sense of sweat smell improved by about $63 \%$ after the last laser session. The frequency of all bacterial strains decreased in the intervention group except Staphylococcus epidermidis which was significant. In the control group, there was no significant decrement in any bacterial strains and even the prevalence of more strains including Staphylococcus aureus and $S$. epidermidis increased. Counting the mean bacterial colon showed a slight decrement of the bacterial count following the laser.

Conclusion: The use of laser radiation, even with the aim of hair removal, can alter the microbial flora, and it can be accompanied by the improvement of the smell of sweat. The effect of the laser on different bacterial strains is quite different, which can depend on the amount of energy, the wavelength, the characteristics of the area under the laser, and also the structural properties of the membrane of the microorganism itself.
\end{abstract}

Keywords: Alexandrite Laser; Microbial Flora; Hair Removal; Axillary odor; Bacterial strains

\section{Introduction}

The existence of a balance between various microorganisms on the skin and the host components such as immunological and barrier systems introduces the skin as a persistent ecosystem. ${ }^{1}$ Although some of these microorganisms are exogenous and harmful for skin and internal organs, some of them play a supportive role as the normal flora on the skin. ${ }^{2}$ Skin flora is composed of aerobic cocci, coryneform bacteria, gram-negative bacteria and yeast (Table 1).

These organisms help to prevent skin infections by providing ecological competition for pathogenic microorganisms and by hydrolyzing the lipids of sebum to produce free fatty acids, which are toxic to many bacteria. ${ }^{4}$ The microbiome of axillary skin is usually dominated by staphylococci or corynebacteria, or occasionally Propionibacteria. ${ }^{5-7}$ The moist environment of the human axilla is characterized by the presence of oily and odorless fluids containing proteins, cholesterol, steroid derivatives, squalene and a wide range of lipids. These substances are secreted by eccrine, apocrine and sebaceous glands. ${ }^{8-10}$ The smell of sweat is the result of the interaction between these substances and the resistant microbiome in the axillary region. Therefore, it seems that the eradication of such skin-related microorganisms can be an applicable method for reducing axillary odor. ${ }^{11-14}$

Laser hair removal is one of the most often used cosmetic procedures ${ }^{15}$ and has been proven to be a superior and more permanent solution for unwanted hair compared to other techniques. ${ }^{16-19}$ The efficacy of certain laser systems at destroying hair follicles within the skin is based on the theory of selective photothermolysis and its further development. ${ }^{20,21}$ This concept aims at the permanent elimination of the dermal papilla and/or the stem cells within the bulge region. Terminal hair follicles are selectively damaged if a wavelength absorbed by melanin 
Table 1. Normal Skin Flora ${ }^{3}$

\begin{tabular}{lll}
\hline Class & Organisms & Location on Body \\
\hline Aerobic cocci & $\begin{array}{l}\text { Staphylococcus aureus, S. saprophyticus, S.epidermidis, } \\
\text { Micrococcus luteus, M. roseus, M. varians }\end{array}$ & All body sites, especially intertriginous areas \\
Aerobic coryneform bacteria & $\begin{array}{l}\text { Corynebacterium minutissimum, C. lipophilicus, C. xerosis, C. } \\
\text { jeikeium, Brevibacterium epidermidis }\end{array}$ & $\begin{array}{l}\text { Intertriginous areas (e.g. axillae, groin, toe } \\
\text { webs) }\end{array}$ \\
Anaerobic coryneform bacteria & Propionibacterium aenes, P. granulosum, P. avidum & Sebaceous glands and follicles \\
Gram-negative bacteria & Acinetobacter spp. & Axillae, perineum, antecubital fossae \\
Yeast & Malassezia furfur & Skin rich in sebaceous glands (e.g. scalp) \\
\hline
\end{tabular}

is applied. ${ }^{16,22}$ Although a number of lasers and non-laser light sources have been developed for the purpose of hair removal, the $755 \mathrm{~nm}$ alexandrite and $800-810 \mathrm{~nm}$ diode lasers are considered as common options for hair removal among individuals with Fitzpatrick skin types I-IV. ${ }^{23,24}$ Since laser hair removal is an elective procedure, pain is an important factor in determining treatment suitability and treatment compliance. It has been observed that intra- and post-operative pain is rated mild to moderate with the alexandrite laser but moderate to severe with the diode laser. ${ }^{25}$ It has already been reported that axillary bromhidrosis and hyperhidrosis improve with laser application. ${ }^{26}$ But these complaints have lately been reported as new side effects of hair removal lasers. ${ }^{27,28}$ The diseases of sweat glands are relatively common and can produce the significant quality of life impairments. Hyperhidrosis is defined as excessive sweat production and has idiopathic or secondary etiologies. Bromhidrosis and chromhidrosis are rare diseases but they can also impact the quality of life as hyperhidrosis. ${ }^{29}$ Coryneform bacteria are the only ones producing the usual odor in the axillary region by the process of apocrine sweat decomposing. Cocci, however, apparently do not have this ability. ${ }^{28}$

It seems that the axillary odor and its related factors have been favorite subjects of different studies. The application of new technologies, especially lasers, has become popular in clinics. Therefore, studies have been done to verify that the different effects of lasers on the human body can reveal unknown aspects of this technology. Based on previous studies, it is still unknown if axillary hair removal with a laser alters the microbial flora of that region. Some studies have investigated the effect of different lasers on bacteria, ${ }^{30-32}$ while no one included the microbial flora of human skin. The present study aimed to determine the microbial flora of the axillary region before and after the third and sixth hair removal alexandrite laser sessions in 30 healthy women. Then comparing the bacterial cultivation results of this group with their matched controls at the same time intervals could determine any differences. Any kinds of interventions in the axillary region of the control group were prohibited in order to have a correct comparison.

\section{Methods}

This study is a quasi-experimental clinical trial on the patients who were seen at the laser clinic of Rasoul-eAkram hospital. The study protocol and all procedures were approved by the ethical committee of human research at Iran University of Medical Sciences. Also, the study was registered in the Iranian Registry of Clinical Trials (identifier: IRCT2015090718210N3). This study happened between February 2016 and August 2017. All participants provided written informed consent. The total number of the participants in this study was 60 women whose ages ranged from 20 to 40 . Thirty women who wanted to do the axillary hair removal laser were chosen as the intervention group and another 30 women who were referred to the laser clinic for other reasons except hair removal were chosen as the control group. Therefore, the selection of the participants in this study was non-random and the inclusion and exclusion criteria were applied to both groups. All potential participants were screened by means of an outpatient visit in the dermatologic clinic of the hospital to meet the exclusion criteria. Women who passed the screening visit were asked to come for future clinic visits. Those who declined or did not appear at the clinic were classified as having declined to participate. To match all the participants in both case and control groups, women with Fitzpatrick skin type III and without any previous laser hair removal or any skin and systemic diseases such as diabetes, psoriasis, atopic dermatitis, herpetic and dermatophyte infection and taking immunosuppressive drugs were considered as the eligible group of this study. The participants who had consumed any kind of oral or topical antibiotics within 72 hours before any sampling session were removed from the study. The eligible women were asked to avoid using deodorants, antifungal or antibacterial cleansing products in their axillary regions throughout the study. They were also asked to remove their axillary hairs only by shaving and to wear cotton-made underwear. In addition, they were asked not to wash that region for 24 hours before the laser. It should be noted that with disregarding one participant to continue the procedure, another participant was substituted.

The used laser device for this study was the GentleMax Pro alexandrite laser (Candela Corp., Boston, MA) with 
a wavelength of $755 \mathrm{~nm}$, adjustable pulse duration of 3 to 100 milliseconds, fluence of 6 to $20 \mathrm{~J} / \mathrm{cm}^{2}$, and spot size of 6 to $18 \mathrm{~mm}$. The candela laser uses a cryogen spray system (DCD) to prevent epidermal damage. All cases got 6 treatment sessions with an interval of 4 to 6 weeks. Laser parameters for the first 3 sessions were $18 \mathrm{~mm}$ spot size, 3 $\mathrm{ms}$ pulse duration, and $10 \mathrm{~J} / \mathrm{cm}^{2}$ fluence and for the last 3 sessions, fluence changed to $14 \mathrm{~J} / \mathrm{cm}^{2}$. It should be noted that no topical or local anesthesia was administered along with the laser treatment. All cases were also sent to the antimicrobial research center of the hospital for microbial flora diagnosis before the intervention and after the third and sixth sessions. The control group was sent for sampling to the same laboratory without any interventions on the first visit and 3 and 6 months thereafter. To keep the microbiologist blind on identity the samples' group, they were taken by the laboratory technicians in the first place and then a number was assigned to each sample and then delivered to the microbiologist.

Methodologically, the swabs impregnated with sterile normal saline were pulled 3 times and clockwise on the right axillary site, and the samples were placed on a medium containing blood agar, nutrient agar, and EMB (eosin methylene blue) agar and then incubated at $37^{\circ} \mathrm{C}$ for 24 hours. After performing Gram staining and detecting the Gram-positive or negative strains, additional experiments were carried out, including exclusive cultivation, diagnostic disks, and biochemical tests. In this study, all bacteria were gram-positive. Catalase and oxidase tests were performed on the bacteria to differentiate Staphylococcus from Streptococcus and Micrococcus from Staphylococcus respectively. Then catalase-positive bacteria were transferred to a mannitol salt agar medium and a coagulase test was performed to detect Staphylococcus aureus. The used antibiotic disks included novobiocin, furazolidone, and bacitracin disks, and a zone of inhibition greater than $16 \mathrm{~mm}, 9$ $\mathrm{mm}$ and $9 \mathrm{~mm}$ in diameter has indicated susceptibility to these antibiotics respectively. ${ }^{33-35}$ The diagnostic disk results are illustrated in Figure 1, in which $(a)$ shows the susceptibility of Staphylococcus to novobiocin and furazolidone disks and resistance to the bacitracin disk, $(b)$ shows the susceptibility of Staphylococcus saprophyticus to the furazolidone disk and resistance to bacitracin and novobiocin disks, and (c) shows the susceptibility of Micrococcus luteus to bacitracin and novobiocin disks and

Staphylococcus

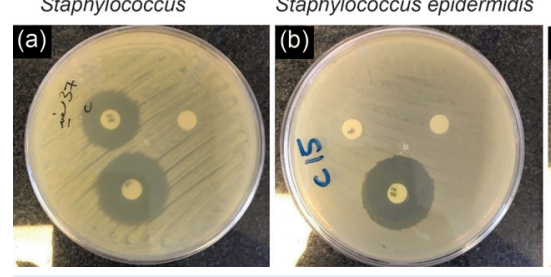

Figure 1. Results of Diagnostic Disks. resistance to the furazolidone disk.

For colony counting, the standard plate count method was performed. For this purpose, different dilutions of the bacteria were prepared, and each dilution was taken on a plate containing a culture medium. The best dilution that is required for bacterial counting is actually a plate containing 30 to 300 colonies. ${ }^{33-38}$ Fewer than 30 colonies are not acceptable for statistical reasons (too few may not be representative of the sample) and more than 300 colonies on a plate are likely to produce colonies too close to each other to be distinguished as distinct colonyforming units. ${ }^{38-40}$ The suspension was made from the bacterium and then prepared 10 tubes which containing $9 \mathrm{ml}$ distilled sterile water including bacteria in different dilutions from $10^{-1}$ to $10^{-10}$. Then, $10 \mu \mathrm{m}$ of each dilution (bacterial concentration) on the nonselective agar plates were incubated at $37^{\circ} \mathrm{C}$ for 24 hours. In this regard, the colony-forming unit was calculated as the number of colonies on the plate counted in the reciprocal of the dilution of the sample. ${ }^{33-38}$

The assessment of the sweat smell was performed by dividing the sweat smell into 3 different categories, namely better, worse and unchanged. The participants were asked to report changes in their sweat smell at the end of the study regarding the 3 mentioned categories.

The results were presented as mean \pm standard deviation (SD) for the quantitative variables and were summarized by absolute frequencies and percentages for the categorical variables. The quantitative variables with a normal distribution were compared using the t-test and with abnormal distribution by the Mann-Whitney test. Also, the categorical variables were compared using the chi-square test or Fisher exact test. For the statistical analysis, the SPSS software version 16.0 for windows (SPSS Inc., Chicago, IL) was used. The $P$ value of 0.05 or less was considered statistically significant.

\section{Results}

The mean age of the case and the control groups were $29.77 \pm 4.35$ years and $31.03 \pm 4.35$ years respectively, which did not show any difference between both groups $(P=0.264)$. In the intervention group, the counted mean bacterial colony before the laser was $17.97 \times 10^{6}$, which was reduced to $17.72 \times 10^{6}$ after the third session and to $17.26 \times 10^{6}$ after the sixth session of the hair removal laser. The results indicated a slight decrement trend in bacterial counting following laser exposure $(P=0.001)$. Considering the age variable as a confounding factor, the bacterial colony count continued to be the same $(P=0.001)$. The smell of sweat improved in 19 cases $(63.3 \%)$, remained unchanged in 6 cases $(20 \%)$, and was exacerbated in residual 5 cases $(16.7 \%)$ based on their own concept (Figure 2). All thirty controls reported no change in their sweat smell.

In correlation between the sweet odor and the bacterial 


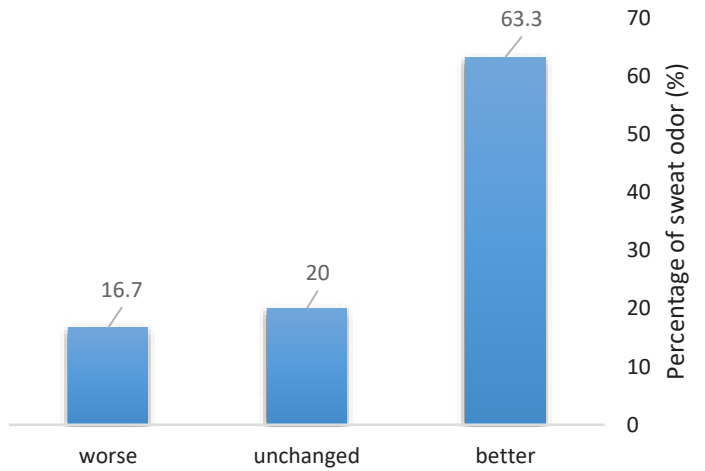

Figure 2. Percentage of Sweat Odor Changes After the Completion of Laser Sessions.

strains after the last laser session, the prominent bacterial strains in better, worse and unchanged categories were Staphylococcus epidermidis, M. luteus, and S. aureus respectively (Figure 3 ).

In the intervention group, before the laser, the most cultivated strains were $S$. aureus alone and the lowest was Staphylococcus saprophyticus alone $(33.3 \%$ and $6.7 \%$ respectively) (Figure 4 ). After the third session of the laser, the highest frequency was related to $S$. aureus alone and the lowest frequency was related to M. luteus with S. epidermidis (Figure 4), and after the sixth session, the highest frequency was related to $S$. epidermidis and the lowest was associated with $S$. aureus with $M$. luteus (Figure 4).

Regarding the frequency of each type of bacteria before as well as after the $3^{\text {rd }}$ and $6^{\text {th }}$ laser sessions (Table 2), the results indicated a decrement in the frequency of $M$. luteus with or without $S$. aureus and $S$. aureus alone, while the frequency of $S$. epidermidis significantly increased following the laser. Accordingly, in the control group, there was no significant reduction in the bacterial strains and even there was an increment in the frequency of more strains including $S$. aureus and S. epidermidis, $M$. luteus, and Staphylococcus saprophyticus (Figure 5). Finally, there was no difference between both groups in terms of the frequency of different bacterial strains before the laser (Table 2). However, after the third laser

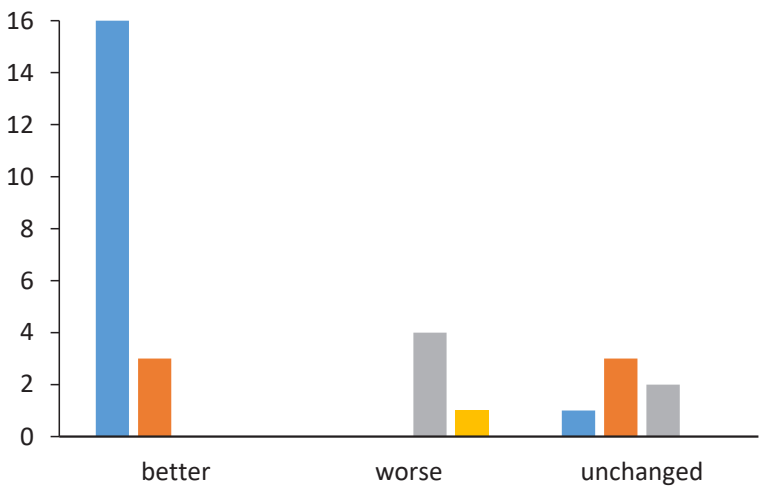

$\square$ S. epidermidis $\square$ S. aureous $\square$ M. luteus $\square$ M.luteus/S.aureous

Figure 3. Correlation Between the Sweet Odor and the Bacterial Strains After the Last Laser Session.

session, it was revealed that the lower frequency of $M$. luteus with $S$. aureus (6.7\% versus $30 \%)$ as well as $M$. luteus with Staphylococcus saprophyticus ( $0 \%$ versus $10 \%)$ occurred in the intervention group $(P=0.007)$. Also, the prevalence of $S$. aureus strains in the intervention group was significantly more than that in the control group (30\% versus $20 \%)(P=0.017)$. However, after the sixth laser treatment, a significantly higher frequency of S. epidermidis was observed in the intervention group compared to the control group (57.6\% versus $26.7 \%)$ $(P=0.001)$.

\section{Discussion}

No direct correlations were observed between odor precursor concentration in underarms and sweat odors, which underlines the importance of bacteria in odor formation. ${ }^{41}$ Therefore, the effect of using laser hair removal on the axillary microbial flora was studied in the present research. Culture-based methods have provided the basis for studying the correlation between bacterial communities and body odors. The microbiome of the axillary skin is usually dominated by staphylococci or corynebacteria, or occasionally propionibacteria. ${ }^{5-7,41}$ This study found that Staphylococcus spp. was the predominant

Table 2. The Change in the Bacterial Strains of the Intervention Group Versus the Control Group Before and After the 3rd And 6th Sessions

\begin{tabular}{|c|c|c|c|c|c|c|}
\hline \multirow{2}{*}{ Bacterial Strains } & \multicolumn{2}{|c|}{ Before Laser } & \multicolumn{2}{|c|}{ After Third Session } & \multicolumn{2}{|c|}{ After Sixth Session } \\
\hline & Laser Group & Control Group & Laser Group & Control Group & Laser Group & Control Group \\
\hline Micrococcus luteus & $5(16.7 \%)$ & $3(10.0 \%)$ & $7(23.3 \%)$ & $5(16.7 \%)$ & $6(20.0 \%)$ & $5(16.7 \%)$ \\
\hline Micrococcus luteus/Staphylococcus aureus & $3(10.0 \%)$ & $7(23.3 \%)$ & $2(6.7 \%)$ & $9(30.0 \%)$ & $1(3.3 \%)$ & $2(6.7 \%)$ \\
\hline Micrococcus luteus/Staphylococcus epidermidis & $5(16.7 \%)$ & $0(0.0 \%)$ & $1(3.3 \%)$ & $0(0.0 \%)$ & $0(0.0 \%)$ & $0(0.0 \%)$ \\
\hline Micrococcus luteus/Staphylococcus saprophyticus & $0(0.0 \%)$ & $4(13.3 \%)$ & $0(0.0 \%)$ & $3(10.0 \%)$ & $0(0.0 \%)$ & $2(6.7 \%)$ \\
\hline Staphylococcus aureus & $10(33.3 \%)$ & $10(33.3 \%)$ & $9(30.0 \%)$ & $6(20.0 \%)$ & $6(20.0 \%)$ & $12(40.0 \%)$ \\
\hline Staphylococcus epidermidis & $5(16.7 \%)$ & $6(20.0 \%)$ & $8(26.7 \%)$ & $7(23.3 \%)$ & $17(56.7 \%)$ & $8(26.7 \%)$ \\
\hline Staphylococcus saprophyticus & $2(6.7 \%)$ & $0(0.0 \%)$ & $3(10.0 \%)$ & $0(0.0 \%)$ & $0(0.0 \%)$ & $1(3.3 \%)$ \\
\hline
\end{tabular}




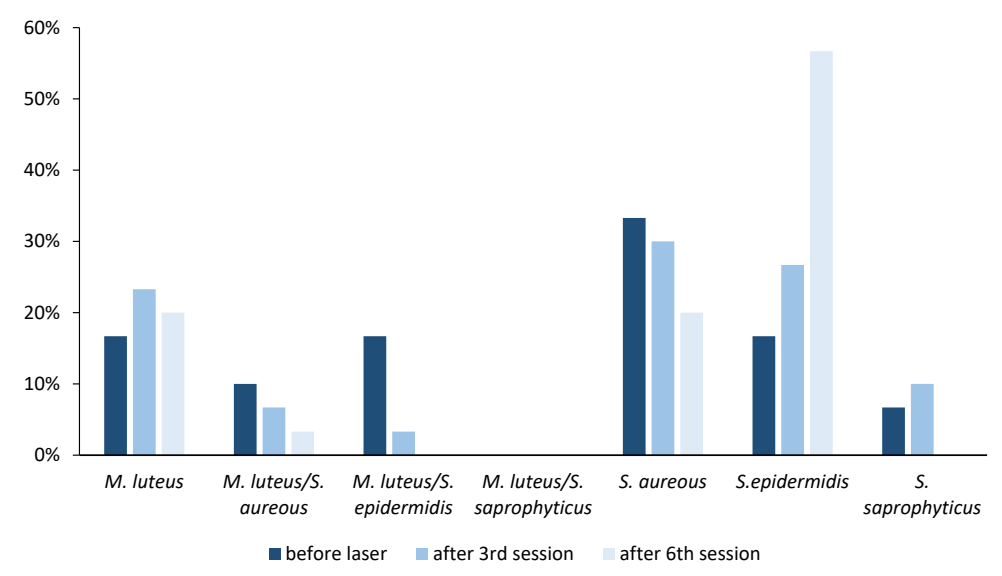

Figure 4. Frequencies of the Bacterial Strains in Different Intervals Within the Intervention Group.

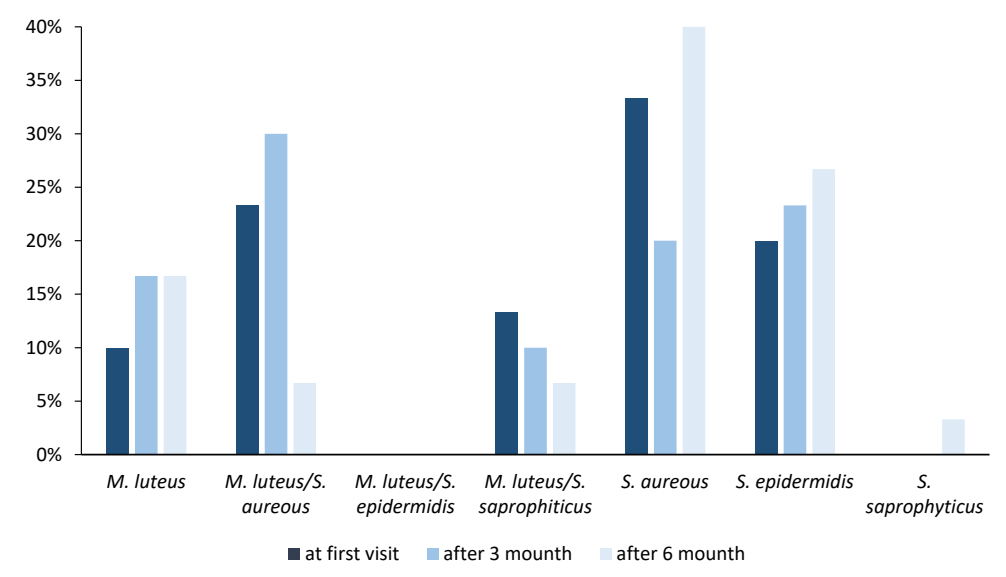

Figure 5. Frequencies of Bacterial Strains in Different Intervals Within the Control Group.

bacterium in the cultivation of the axillary region before any intervention in both case and control groups. This result is supported by culture-based ${ }^{5}$ as well as molecularbased studies. ${ }^{7}$ Callewaert et $\mathrm{al}^{7}$ showed that females predominantly clustered within the Staphylococcus cluster, whereas males clustered more in the Corynebacterium cluster. Skin anatomical and physiological differences, as in hair growth, skin thickness, hormone, sweat and sebum production, form the basis for these microbial differences. ${ }^{10}$ All participants in our study were female with Staphylococcus spp. as a dominant resident of their axilla. The gram-negative bacteria and coryneform bacteria were not detected at all. Most participants mentioned their sweat smell became better at the end of the laser sessions. It should be noted that the effect of laser therapy on various bacterial strains was quite different so that some bacterial strains (such as $M$. luteus and $S$. aureus) were more affected by laser radiation, while some strains, such as $S$. epidermidis, received the least effect by laser therapies, and even an increase in the colony of this strain might occur. Few similar studies were performed in animal models. Nussbaum et al showed that the normal skin flora of the rat's wound was reduced by the use of light at $808 \mathrm{~nm}$, which subsequently increased the presence of $S$. aureus. In fact, it has not become clear that reduction in normal flora caused increasing $S$. aureus, or laser light directly stimulates their growth. ${ }^{30}$ Manolis et al examined the effect of the carbon dioxide laser and mechanical abrasion on skin microbial flora of rats. The reduction of the post-laser microbial count was observed in diphtheroid and staphylococci in comparison to the pre-laser and post-abrasion specimens which could be a positive clinical outcome of the skin resurfacing lasers. ${ }^{31}$ Although these studies ${ }^{30,31}$ could show a decrement in microbial counts in animal models, the mean bacterial colony counts didn't show a significant change during laser therapy in this study. The laser wavelength which was used in those two studies was greater than that in this study. Pereira et al investigated the effect of $904 \mathrm{~nm}$ laser radiation in the in vitro bacterial culture and in vivo infected wounds of the rats. They found out that laser therapy could not prevent $S$. aureus growth on a blood agar medium but had a relative preventative effect on the $S$. aureus infected wounds. ${ }^{32}$ It seems that the different 
wavelengths of the laser, the characteristics of the area where the laser radiates (intact skin or wound), and the structural composition of bacteria determined the effects of the laser on the bacteria and thereby prevented or even stimulated their growth.

\section{Conclusion}

It can be said that the use of laser radiation, even with the aim of hair removal, can alter the microbial flora, and as a result, it can be accompanied by the improvement of the smell of sweat. The effect of the laser on different bacterial strains is quite different, which can depend on the amount of the energy, the wavelength, the characteristics of the area under the laser, and also the structural properties of the membrane of the microorganism itself. It is not exactly known that the laser light independently increases the growth of a specific bacterial strain or its growth inhibitor effect on other strains has created better conditions for the growth of that specific strain. On the other hand, different responses to laser light in different species of bacteria are due to mutation in their genome or the result of the changes in their membrane. It seems that further evaluations are needed for a clear reflection of all the aspects of the laser impact on bacteria. In this field, culture-independent analyses of bacterial communities like molecular analysis, allowing the determination of any changes in the ultra-structural parts of bacteria (e.g., genome and proteins). Therefore, these types of studies can show which part of the bacterial structure is affected by the laser. It is also suggested that laser-based studies be conducted in a larger group including women and men. Also, a post-laser procedure follow-up with longer intervals could determine the microbial and sweet odor changes.

\section{Ethical Considerations}

This study has been approved by the ethical committee of Iran University of Medical Sciences.

\section{Conflict of Interests}

The authors declare that they have no conflict of interest.

\section{Funding}

This study was funded by the Deputy of Research \& Technology of Iran University of Medical Sciences (Grant Number: 26283-134-02-94).

\section{Acknowledgments}

Authors would like to thank H. Bahari, M. Nikja and G. Mahdavi, the Laser Clinic staff, for their assistance with this study.

\section{References}

1. Grice EA. The intersection of microbiome and host at the skin interface: genomic- and metagenomic-based insights. Genome Res. 2015;25(10):1514-20. doi: 10.1101/ gr.191320.115.

2. Roth RR, James WD. Microbial ecology of the skin. Annu Rev Microbiol. 1988;42:441-64. doi:10.1146/annurev. mi.42.100188.002301.

3. Bolognia JL, Jorizzo JL, Schaffer JV. Dermatology. 3rd ed. London: Elsevier Saunders; 2012.

4. Noble WC. Skin bacteriology and the role of Staphylococcus aureus in infection. Br J Dermatol. 1998;139(Suppl 53):912. doi:10.1046/j.1365-2133.1998.1390s3009.x.

5. Leyden JJ, McGinley KJ, Hölzle E, Labows JN, Kligman AM. The microbiology of the human axilla and its relationship to axillary odor. J Invest Dermatol. 1981;77(5):413-416. doi:10.1111/1523-1747.ep12494624.

6. Taylor D, Daulby A, Grimshaw S, James G, Mercer J, Vaziri S. Characterization of the microflora of the human axilla. Int J Cosmet Sci. 2003;25(3):137-145. doi:10.1046/j.14672494.2003.00181.x.

7. Callewaert C, Kerckhof FM, Granitsiotis MS, Van Gele M, Van de Wiele T, Boon N. Characterization of Staphylococcus and Corynebacterium clusters in the human axillary region. PLoS One. 2013;8(8):e70538. doi: 10.1371/journal.pone.0070538.

8. Labows JN, Preti G, Hoelzle E, Leyden J, Kligman A. Steroid analysis of human apocrine secretion. Steroids. 1979;34(3):249-258. doi:10.1016/0039-128x(79)90077-1.

9. Greene RS, Downing DT, Pochi PE, Strauss JS. Anatomical variation in the amount and composition of human skin surface lipid. J Invest Dermatol. 1970;54(3):240-247. doi:10.1111/1523-1747.ep12280318.

10. Wilke K, Martin A, Terstegen L, Biel SS. A short history of sweat gland biology. Int J Cosmet Sci. 2007;29(3):169-179. doi:10.1111/j.1467-2494.2007.00387.x.

11. James AG, Austin CJ, Cox DS, Taylor D, Calvert R. Microbiological and biochemical origins of human axillary odour. FEMS Microbiol Ecol. 2013;83(3):527-540. doi:10.1111/1574-6941.12054.

12. Barzantny H, Brune I, Tauch A. Molecular basis of human body odour formation: insights deduced from corynebacterial genome sequences. Int J Cosmetic Sci. 2012;34(1):2-11. doi:10.1111/j.1468-2494.2011.00669.x.

13. Schommer NN, Gallo RL. Structure and function of the human skin microbiome. Trends Microbiol. 2013;21(12):660-668. doi:10.1016/J.TIM.2013.10.001.

14. Rieg S, Seeber S, Steffen H, Humeny A, Kalbacher H, Stevanovic S, et al. Generation of multiple stable dermcidinderived antimicrobial peptides in sweat of different body sites. J Invest Dermatol. 2006;126(2):354-365. doi:10.1038/ sj.jid. 5700041 .

15. Ibrahimi OA, Avram MM, Hanke CW, Kilmer SL, Anderson RR. Laser hair removal. Dermatol Ther. 2011;24(1):94-107. doi:10.1111/j.1529-8019.2010.01382.x.

16. Dierickx CC, Grossman MC, Farinelli WA, Anderson RR. Permanent hair removal by normal-mode ruby laser. Arch Dermatol. 1998;134(7):837-842. doi:10.1001/ archderm.134.7.837.

17. Görgü M, Aslan G, Aköz T, Erdoğan B. Comparison of alexandrite laser and electrolysis for hair removal. Dermatol Surg. 2000;26(1):37-41. doi:10.1046/j.15244725.2000.99104.x

18. Lou WW, Quintana AT, Geronemus RG, Grossman MC. Prospective study of hair reduction by diode laser (800nm) with long-term follow-up. Dermatol Surg. 2000;26(5):428432. doi:10.1046/j.1524-4725.2000.99260.x. 
19. Fayne RA, Perper M, Eber AE, Aldahan AS, Nouri K. Laser and light treatments for hair reduction in Fitzpatrick skin types IV-VI: A comprehensive review of the literature. Am J Clin Dermatol. 2018;19(2):237-52. doi: 10.1007/s40257017-0316-7.

20. Anderson RR, Parrish JA. Selective photothermolysis: precise microsurgery by selective absorption of pulsed radiation. Science. 1983;220(4596):524-527. doi:10.1126/ science.6836297.

21. Altshuler GB, Anderson RR, Manstein D, Zenzie $\mathrm{HH}$, Smirnov MZ. Extended theory of selective photothermolysis. Lasers Surg Med. 2001;29(5):416-432. doi:10.1002/lsm.1136.

22. Greveling K, Prens EP, Liu L, van Doorn MBA. Noninvasive anaesthetic methods for dermatological laser procedures: a systematic review. J Eur Acad Dermatol Venereol. 2017;31(7):1096-1110. doi:10.1111/jdv.14130.

23. Haedersdal M, Wulf HC. Evidence-based review of hair removal using lasers and light sources. J Eur Acad Dermatol Venereol. 2006;20(1):9-20. doi:10.1111/j.14683083.2005.01327.x.

24. Grunewald S, Bodendorf MO, Zygouris A, Simon JC, Paasch U. Long-term efficacy of linear-scanning 808nm diode laser for hair removal compared to a scanned alexandrite laser. Lasers Surg Med. 2014;46(1):13-19. doi:10.1002/lsm.22185.

25. Handrick C, Alster TS. Comparison of long-pulsed diode and long-pulsed alexandrite lasers for hair removal: a longterm and histologic study. Dermatol Surg. 2001;27(7):622626. doi:10.1046/j.1524-4725.2001.00338.x.

26. Jung SK, Jang HW, Kim HJ, Lee SG, Lee KG, Kim SY, et al. A prospective, long-term follow-up study of 1,444 nm Nd:YAG laser: A new modality for treating axillary bromhidrosis. Ann Dermatol. 2014;26(2):184-188. doi:10.5021/ad.2014.26.2.184.

27. Helou J, Haber R, Kechichian E, Tomb R. A case of generalized bromhidrosis following whole-body depilatory laser. J Cosmet Laser Ther. 2015;17(6):318-320. doi:10.3109 /14764172.2015.1027232.

28. Hélou J, Soutou B, Jamous R, Tomb R. Novel adverse effects of laser-assisted axillary hair removal. Ann Dermatol Venereol. 2009;136(6-7):495-500. doi:10.1016/j. annder.2009.04.005.

29. Semkova K, Gergovska M, Kazandjieva J, Tsankov N. Hyperhidrosis, bromhidrosis, and chromhidrosis: Fold (intertriginous) dermatoses. Clin Dermatol.2015;33(4):483491. doi:10.1016/j.clindermatol.2015.04.013.
30. Nussbaum EL, Mazzulli T, Pritzker KP, Heras FL, Jing F, Lilge L. Effects of low intensity laser irradiation during healing of skin lesions in the rat. Lasers Surg Med. 2009;41(5):372-381. doi:10.1002/lsm.20769.

31. Manolis EN, Tsakris A, Kaklamanos I, Markogiannakis A, Siomos K. In vivo effect of carbon dioxide laser skin resurfacing and mechanical abrasion on the skin's microbial flora in an animal model. Dermatol surg. 2006;32(3):359364. doi:10.1111/j.1524-4725.2006.32073.x.

32. Pereira PR, de Paula JB, Cielinski J, Pilonetto M, Von Bahten LC. Effects of low intensity laser in in vitro bacterial culture and in vivo infected wounds. Rev Col Bras Cir. 2014;41(1):49-55. doi:10.1590/s0100-69912014000100010.

33. Johnson TR, Case CL. Laboratory Experiments in Microbiology. 11th ed. NY: Pearson; 2015.

34. Goldman E, Green LH, editors. Practical Handbook of Microbiology. 2nd ed. Boca Raton, Florida: CRC Press; 2008.

35. Murray PR, Baron EJ, Jorgensen JH, Landry ML, Pfaller MA. Manual of Clinical Microbiology. 9th ed. Washington, D.C.: ASM Press; 2007.

36. Hedges AJ. Estimating the precision of serial dilutions and viable bacterial counts. Int J Food Microbiol. 2002;76(3):207214. doi.org/10.1016/S0168-1605(02)00022-3.

37. JettBD, Hatter KL, Huycke MM, Gilmore MS. Simplified agar plate method for quantifying viable bacteria. Biotechniques. 1997;23(4):648-650. doi:10.2144/97234bm22.

38. Boczek LA, Rice EW, Johnson CH. Total viable counts: Pour plate technique. In: Messer JM, Rice EW, Johnson CH, eds. Encyclopedia of food microbiology. 2nd ed. Elsevier; 2014. doi:10.1016/B978-0-12-384730-0.00330-X

39. Thomas P, Sekhar AC, Mujawar MM. Nonrecovery of varying proportions of viable bacteria during spread plating governed by the extent of spreader usage and proposal for an alternate spotting-spreading approach to maximize the CFU. J Appl Microbiol. 2012;113(2):339-350. doi:10.1111/ j.1365-2672.2012.05327.x

40. Thomas P, Mujawar MM, Sekhar AC, Upreti R. Physical impaction injury effects on bacterial cells during spread plating influenced by cell characteristics of the organisms. J Appl Microbiol. 2014;116(4):911-922. doi:10.1111/ jam.12412.

41. Troccaz M, Gaïa N, Beccucci S, Schrenzel J, Cayeux I, Starkenmann C, et al. Mapping axillary microbiota responsible for body odours using a culture-independent approach. Microbiome. 2015:24;3(1):3. doi: 10.1186/ s40168-014-0064-3. 